\title{
On the Evolutionary Status of the WR-type PNN
}

\section{R. Tylenda and S.K. Górny}

Copernicus Astronomical Center, Toruń, Poland

Some observed parameters of planetary nebulae with H-deficient (mostly WR-type) nuclei (PNN) are compared to those of $\mathrm{H}$-rich ones and to theoretical predictions from the born again scenario. An example is shown in Fig. 1

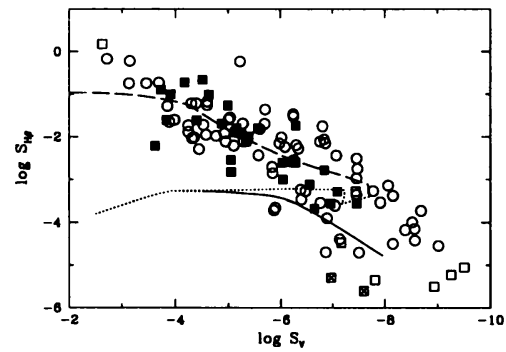

Figure 1: $S_{\mathrm{H} \beta}-S_{\mathrm{V}}$ diagram. $S_{\mathrm{H} \beta}-$ surface brightness of the nebula in $\mathrm{H} \beta, S_{\mathrm{V}}=F_{\mathrm{V}} /\left(\pi \theta^{2}\right)$, $F_{\mathrm{V}}$ - stellar flux in the $\mathrm{V}$ band, $\theta$ - nebular angular radius. Circles: $\mathrm{H}$-rich $\mathrm{PNN}$, squares: $\mathrm{H}-$ deficient PNN (filled squares: WR-type PNN, crossed squares: A 30 and A 78). The curve shows the evolution of a model with a late He-shell flash (see text).

The H-deficient PNN occupy practically the same region in Fig. 1 as the H-rich ones. $S_{\mathrm{H} \beta}$ is a measure of the expansion stage of the nebula while $S_{\mathrm{V}}$ informs on the evolutionary stage of the central star. Therefore Fig. 1 suggests that the evolutionary status (time elapsed since leaving the AGB, evolutionary rate in the PN phase) is similar for both groups of objects. This seems to imply that the $\mathrm{H}$-deficient PNN arrive directly from the AGB and confirms the conclusion of Górny \& Stasińska (1995) that there is no systematic difference in mass between the $\mathrm{H}$-deficient and the $\mathrm{H}$-rich PNN.

Some authors suggest that the $\mathrm{H}$-deficient PNN are born again nuclei after a late helium shell flash. The curve in Fig. 1 shows the expected evolution of a nebula with a flashing PNN (based on Iben at al. 1983). The model PNN ionizes a $0.2 \mathrm{M}_{\odot}$ nebula expanding at $20 \mathrm{~km} / \mathrm{s}$. Dashed curve shows the initial evolution during the $\mathrm{H}$-burning phase. Dotted curve shows the fast return of the PNN towards the AGB caused by the onset of a late $\mathrm{He}-$ shell flash. The final evolution as a born again, He-burning, $\mathrm{H}-$ deficient PNN is displayed by a full curve.

As can be seen from Fig. 1 the born again nuclei are expected to appear at positions significantly shifted in respect to the H-burning PNN. This is in contradiction with the observations. Thus we conclude that most of the H-deficient PNN, especially the WRtype PNN, are not born again central stars. They presumably originate directly from the AGB. Note, however, that positions of a few H-deficient PNN, including A 30 and A 78, in Fig. 1 are compatible with the predictions of the born again model.

Acknowledgments: This work was supported from KBN grant 2.P03D.027.10

\section{REFERENCES}

Górny S.K., Stasińska G., 1995, A\&A 303, 893

Iben I.,Jr., Kaler J.B., Truran J.W., Renzini A., 1983, ApJ 264, 605 